\title{
Nonalcoholic Fatty Liver Disease and Obesity
}

\author{
Arka De $^{1}$, Ajay Duseja ${ }^{2}$
}

\begin{abstract}
The ability of the body to store energy in adipose tissue for utilization during exigency has helped us cope with periodic food shortages through much of human history. The easy availability of calorie-dense food and a sedentary lifestyle has, however, resulted in this becoming a bane with adverse health consequences. Both obesity and nonalcoholic fatty liver disease (NAFLD) are lifestyle diseases that have emerged as substantial public health problems globally and in India. Obesity is an important risk factor for NAFLD and its severe form of nonalcoholic steatohepatitis (NASH). Weight loss is the only proven beneficial intervention in NAFLD/NASH. This provides a unique opportunity for managing NASH in obese patients. Lifestyle interventions including dietary modifications and exercise should be the primary modality for weight loss and have shown to be feasible and effective in obese patients with NASH. Although not yet indicated for the management of NASH per se, anti-obesity drugs and bariatric procedures may be considered in selected patients if otherwise indicated and may lead to indirect hepatic benefits secondary to weight loss.
\end{abstract}

Keywords: Fatty liver, Hepatic steatosis, Metabolic dysfunction-associated fatty liver disease, Metabolic syndrome, Nonalcoholic fatty liver disease, Nonalcoholic steatohepatitis.

Journal of Postgraduate Medicine, Education and Research (2021): 10.5005/jp-journals-10028-1430

\section{INTRODUCTION}

For much of human history, food supplies have been scarce and often intermittent. Adipose tissue acts as a storehouse of excess energy in the form of triglycerides which can be broken down to free fatty acids when required with the release of the stored energy. This ability to store energy that is not immediately required and reuse it in times of food shortage was an essential survival strategy in human evolution. The easy availability of food coupled with sedentary lifestyles and physical inactivity in modern times has resulted in this unique evolutionary strategy becoming a bane with adverse health-related outcomes. ${ }^{1}$

Obesity may be considered to be a state of excessive adipose tissue deposition which is often accompanied by chronic, low-grade inflammation. ${ }^{1}$ Nonalcoholic fatty liver disease (NAFLD) refers to the excessive accumulation of fat in the hepatocytes (>5\%) in the absence of significant alcohol intake or other cause of hepatic steatosis. ${ }^{2}$ Both these lifestyle diseases have become significant public health problems in the 21st century. ${ }^{1,3}$ There is an intrinsic link between obesity and NAFLD. Not only is obesity one of the commonest risk factors for NAFLD, but obese patients are also more likely to have nonalcoholic steatohepatitis (NASH) which is usually considered to be the progressive form of NAFLD and has a higher propensity to progress to NASH-related cirrhosis and NASH-related hepatocellular carcinoma (HCC). ${ }^{4-6}$ Furthermore, NAFLD patients who continue to gain weight have a higher risk for progression of fibrosis. ${ }^{7}$ Moreover, reduction in body weight is the only definitively proven intervention in NAFLD. ${ }^{2}$

\section{Nonalcoholic Fatty Liver Disease or Metabolic Dysfunction-associated Fatty Liver Disease}

One of the caveats in the study of NAFLD is that there are no positive biomarkers for diagnosing NAFLD which remains a diagnosis of exclusion. To circumvent this problem, a team of experts has recently proposed the new term metabolic dysfunction-associated
1,2 Department of Hepatology, Postgraduate Institute of Medical Education and Research, Chandigarh, India

Corresponding Author: Ajay Duseja, Department of Hepatology, Postgraduate Institute of Medical Education and Research, Chandigarh, India, Phone: +91-172-2754793, e-mail: ajayduseja@yahoo.co.in

How to cite this article: De A, Duseja A. Nonalcoholic Fatty Liver Disease and Obesity. J Postgrad Med Edu Res 2021;55(1):27-35.

Source of support: Nil

Conflict of interest: None

fatty liver disease (MAFLD) to describe the condition of hepatic steatosis in the presence of metabolic risk factors. ${ }^{8}$ This definition recognizes the intrinsic link between metabolic risk factors like obesity and NAFLD. It also acknowledges that multiple etiologies of the fatty liver may be present in the same patient. For instance, an obese patient with fatty liver may also be consuming significant amounts of alcohol. This would have precluded the diagnosis of NAFLD. Thus, using the new nomenclature, the patient may be considered to have both MAFLD and alcoholic liver disease. However, several problems need to be sorted out before this new nomenclature is ready for prime time. Most importantly, NAFLD is an umbrella term that refers to a histological spectrum ranging from nonalcoholic fatty liver (NAFL) to NASH, cirrhosis, and HCC. Nonalcoholic fatty liver is usually considered to be relatively innocuous while NASH is considered to be the progressive form of the disease. The new nomenclature of MAFLD does not incorporate this spectrum and all patients with NAFLD may not even have associated metabolic risk factors. Hence, the change in nomenclature looks premature and may even be potentially misleading and confusing for the hepatologists and other specialties. ${ }^{9}$ Hence, in this article, we will be using the term NAFLD rather than MAFLD. Furthermore, in this review, we will explore the epidemiological relationship of obesity and NAFLD, the pathomechanistic links between the two conditions, and discuss the approach to diagnosis and management of these patients. 


\section{Epidemiology of Obesity and Non- alcoholic Fatty Liver Disease: Two Parallel Epidemics}

Increased urbanization, sedentary lifestyle, and easy access to calorie-dense food underlie the parallel upsurge in obesity and NAFLD in recent times. ${ }^{10}$ Obesity was declared to be a global epidemic in 2005 by the World Health Organization. Worldwide, it is estimated that there are 650 million obese individuals with an additional 1.9 billion overweight individuals. ${ }^{11}$ In 2014, the global age-standardized prevalence of obesity among adults was estimated to be $10.8 \%$ in males and $14.9 \%$ in females. ${ }^{12}$ The prevalence of obesity has nearly tripled over the past four decades. Trends suggest that by 2030, almost two in five individuals will be overweight or obese..$^{13} \mathrm{~A}$ substantial proportion of this recent upward trend is accounted for by Asian countries like India and China where historically, obesity was uncommon. ${ }^{14}$ In 1975, there were only 4 lakh obese individuals in India compared with 9.8 crore individuals with obesity in $2014 .{ }^{15}$ The Indian Council of Medical Research estimates that the prevalence of generalized and abdominal obesity in Indian adults varies from 11.8 to $31.3 \%$ and 18.7 to $36.1 \%$, respectively, with a higher prevalence in urban areas. ${ }^{15}$

A recent meta-analysis including 86 studies with more than 85 lakh patients from 22 countries gives us important insights into the global epidemiology of NAFLD. ${ }^{5}$ Almost a quarter of the world's population may have underlying NAFLD with the prevalence being highest in Middle Eastern and South American countries and lowest in Africa. Approximately $50 \%$ of patients with NAFLD are obese. ${ }^{5}$ Indeed, there is a correlation between the geographic prevalence of NAFLD and established obesity parameters. ${ }^{14}$ Among obese patients, the prevalence of NAFLD ranges from 60 to $95 \%$, and up to one-third of these patients may have underlying NASH. ${ }^{16}$

The prevalence of NAFLD in India ranges from 9 to $53 \%$ with a rural-urban divide. ${ }^{17,18}$ An Indian position paper on NAFLD estimated that there are at least 25 million patients with NAFLD in the country with a potential risk for significant liver disease. ${ }^{17}$ Some data suggest that Indian patients with NAFLD are less likely to have morbid obesity compared with Western NAFLD patients. ${ }^{19-21}$ However, generalized overweight or obesity based on body mass index (BMI) and central obesity has been reported in 88 and $80 \%$ of patients with NAFLD, respectively. ${ }^{19}$ Early observations suggested that liver histology findings are relatively innocuous in Indian NAFLD patients with mild-moderate degrees of inflammation and fibrosis. However, this has been questioned by recent data showing the presence of histological NASH in $>60 \%$ of patients of biopsy-proven NAFLD with advanced fibrosis in $35 \%$ of patients. ${ }^{22}$

\section{Pathophysiologic Link between Obesity and Nonalcoholic Fatty Liver Disease}

The current understanding of NAFLD pathogenesis involves a multihit hypothesis with a complex interplay of genetic predilection (e.g., PNPLA3 I148M and the TM6SF2 E167K variants), imbalance of calorie intake and expenditure, insulin resistance, dysfunctional and expanded visceral adipose tissue, altered adipokine levels (decreased adiponectin, increased leptin), lipotoxicity of intrahepatic lipids, changes in gut-microbiome, oxidative stress, and activation of innate immunity which ultimately leads to inflammation, hepatocyte apoptosis, and progressive fibrosis. ${ }^{23}$ Insulin resistance is the central common link between obesity and NAFLD.
It is important to appreciate that the expanded adipose tissue in obese patients is not metabolically inactive. Rather, it is dysfunctional with excess release of proinflammatory adipokines like leptin. Leptin receptors are expressed by many cells of the innate immune system including macrophages and eosinophils. Activation of these receptors leads to the release of proinflammatory cytokines like IL- 6 and TNF- $a$ which further leads to the secretion of more leptin, resulting in a vicious cycle. This is accompanied by a decrease in adiponectin which results in decreased $\beta$-oxidation of fatty acids and a worsening of insulin resistance.

Normal insulin-mediated suppression of peripheral lipolysis is impaired in insulin resistance. Lipolysis in the expanded adipose tissue increases the availability of free fatty acids for uptake in the liver. Insulin resistance also increases the expression of hepatic fatty acid translocase which promotes the uptake of free fatty acids by the liver. Increased blood glucose and elevated insulin levels act via sterol regulatory element-binding protein (SREBP-1c) and carbohydrate responsive element-binding protein (ChREBP) to increase de novo hepatic lipogenesis and inhibit fatty acid oxidation. Thus, there is a net increase in intrahepatic fatty acids which are then esterified to triglycerides. Very low-density lipoprotein (VLDL) is the main route for the extrusion of triglycerides from the liver. Although the secretion of triglycerides in VLDL is increased in NAFLD, this cannot keep pace with the increased triglyceride formation. The excess triglycerides accumulate in the hepatocytes as histologically demonstrable steatosis. However, current evidence suggests that this triglyceride may not be the true culprit behind NASH. Intrahepatic triglyceride levels are similar between patients of NAFL and NASH. Rather certain intermediates like ceramides, diacylglycerol, and lysophosphatidylcholine which are produced during the metabolism of increased hepatic fatty acids may account for the bulk of the lipotoxicity. ${ }^{24}$ These intermediates promote insulin resistance by activating protein kinase $C$, mammalian target of rapamycin (mTOR), and nuclear factor kinase $\mathrm{B}(\mathrm{NF}-\mathrm{KB})$, and inhibiting $\mathrm{Akt}^{25}$

With excessive fat accumulation being the common denominator in both obesity and NAFLD, it is easy to construe that "fat" people have "fatty" livers. However, such a notion is a crude oversimplification. It is now recognized that not all obese individuals are metabolically unhealthy. ${ }^{26}$ Similarly, not all lean individuals are metabolically healthy and $10 \%-15 \%$ of patients with NAFLD are lean. ${ }^{27}$ While obesity has been recognized as one of the most important risk factors for NAFLD, the association between NAFLD and metabolically healthy obesity needs further evaluation. ${ }^{28}$

The distribution of body fat may be predictive of metabolic outcomes. Abdominal obesity often described as apple-shaped or android obesity has been associated with poorer cardiovascular outcomes as compared to pear-shaped or gynoid obesity where fat mainly accumulates in the subcutaneous tissues around the hip and thighs. ${ }^{1}$ Despite some evidence to the contrary, visceral adiposity is associated with higher lipolysis and insulin resistance with increased secretion of proinflammatory and fibrogenic mediators when compared with subcutaneous adipose tissue and higher predisposition to NAFLD (Fig. 1)., ${ }^{1,29-31}$

The ectopic deposition of fat in the liver is explained by the adipose tissue expandability hypothesis. ${ }^{32}$ This hypothesis states that the adipose tissue has limited expandability to accumulate new fat stores. Once the capacity of adipose tissue expansion is exhausted, fat accumulates in ectopic sites like the liver and muscles with resultant lipotoxicity and increased insulin resistance. Subcutaneous adipose tissue appears to be the primary site 


\section{$\square$ Subcutaneous adipose tissue}

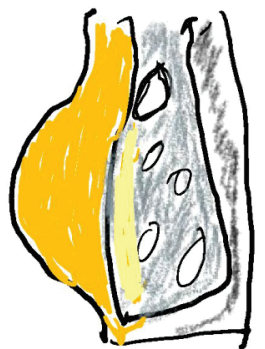

$\square$ Viscera adipose tissue

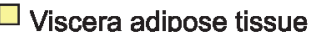

Subcutaneous obesity

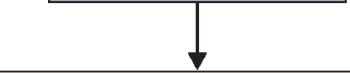

- Normal adipocytes

- M2 macrophages predominate

- Anti-inflammatory adipokines

like adiponectin increased
Anti-inflammatory phenotype with possible protection against metabolic complications and cardiovascular disease

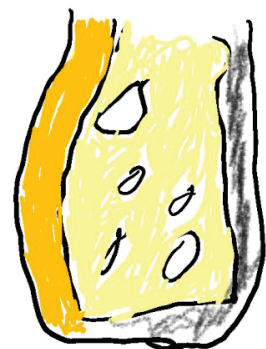

Visceral obesity

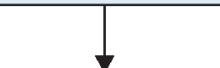

- Dysfunctional adipocytes with apoptosis of asipocytes

- M1 macrophage predominate

- Increased pro-inflammatory cytokines like resistin,

leptin, TNF, IL-6, IL-18 etc

- Decreased anti-inflammatory adipokines

Pro-inflammatory phenotype and insulin resistance with increased risk of diabetes, metabolic syndrome and cardiovascular disease

Fig. 1: Current understanding of subcutaneous and visceral obesity in the pathogenesis of nonalcoholic fatty liver disease/nonalcoholic steatohepatitis

for this initial fat storage as evidenced by its expandability and plasticity while visceral adipose tissue is associated with insulin resistance and poor metabolic outcomes. ${ }^{33}$ On the contrary, some evidence suggests that visceral adipose tissue is only a bystander and subcutaneous adipose tissue may be the main regulator of metabolic health. ${ }^{34,35}$ Whatever may be the case, an inflamed, expanded, and dysfunctional adipose tissue ultimately worsens insulin resistance and predisposes to NAFLD. ${ }^{1}$

\section{Diagnosis of Obesity and Nonalcoholic Fatty Liver Disease}

In Indians, obesity is defined at a BMI $\geq 25 \mathrm{~kg} / \mathrm{m}^{2}$, and those with BMI between $23 \mathrm{~kg} / \mathrm{m}^{2}$ and $25 \mathrm{~kg} / \mathrm{m}^{2}$ are considered as overweight (Table 1). ${ }^{36}$ However, BMI can lead to overestimation of obesity in individuals with a muscular physique. Furthermore, it may not be adequately representative of abdominal obesity. Waist circumference as a surrogate of abdominal obesity is a better predictor of metabolic outcomes. ${ }^{37}$ The cutoff for waist circumference for defining obesity in Indian males and females is $\geq 90$ and $\geq 80 \mathrm{~cm}$, respectively. ${ }^{36}$ In general, both BMI and waist circumference should be routinely measured in all patients. ${ }^{17,37}$ Other methods for assessing adiposity include body impedance analysis, densitometric studies, dual-energy X-ray absorptiometry, and imaging using computed tomography (CT) or magnetic resonance imaging (MRI). CT- or MRI-based assessment has the added advantage of being able to distinguish between visceral and subcutaneous adiposity. It can also simultaneously assess for sarcopenia. However, these techniques are largely limited to research settings and are not routinely required for the evaluation of obesity.
Table 1: Cutoffs for defining overweight, obesity, and central obesity in Indian patients

\begin{tabular}{ll}
\hline Body mass index (BMI) & \\
- Overweight & $23-24.9 \mathrm{~kg} / \mathrm{m}^{2}$ \\
- Obese & $\geq 25 \mathrm{~kg} / \mathrm{m}^{2}$ \\
- Class I & $25-29.9 \mathrm{~kg} / \mathrm{m}^{2}$ \\
- Class II & $\geq 30 \mathrm{~kg} / \mathrm{m}^{2}$ \\
Waist circumference for central obesity \\
- Males & $\geq 90 \mathrm{~cm}$ \\
- Females & $\geq 80 \mathrm{~cm}$ \\
\hline
\end{tabular}

Nonalcoholic fatty liver disease is a diagnosis of exclusion. Diagnosis requires the demonstration of hepatic steatosis in the absence of significant alcohol intake and other etiologies of hepatic steatosis. ${ }^{17}$ Fatty liver is usually detected on ultrasound although this modality lacks sensitivity when the degree of hepatic steatosis is $<30 \%$ and it may be difficult to get a good window in obese patients. ${ }^{38} \mathrm{CT}$ - or MRI-based techniques have higher sensitivity for hepatic steatosis, although they are rarely used for this purpose in clinical practice. Nowadays, fatty liver is often detected and graded based on the controlled attenuation parameter (CAP) that is obtained during vibration controlled transient elastography (VCTE, FibroScan). ${ }^{39}$ An individual patient data meta-analysis of $>2,700$ patients has suggested optimal cutoffs of 248,268 , and $280 \mathrm{~dB} / \mathrm{m}$ for detecting mild, moderate, and severe steatosis, respectively. ${ }^{39}$ Liver biopsy remains the gold standard for differentiating NASH from simple steatosis. ${ }^{2,24}$ However, biopsies may not be feasible in all patients in routine clinical practice and are directed toward patients likely to have NASH with or without significant fibrosis. In general, patients with obesity and metabolic syndrome are considered to be at a higher risk of having NASH. ${ }^{17}$ 
Fibrosis has now been shown to be the main determinant of clinical and histological outcomes in NAFLD. ${ }^{40}$ Fibrosis can be reliably estimated using noninvasive techniques. This includes various proprietary [enhanced liver fibrosis (ELF) test, FibroTest, FibroMeter] and nonproprietary [NAFLD fibrosis score (NFS), ASTplatelet ratio index (APRI), FIB-4] blood-based tests and elastographic techniques. ${ }^{41,42}$ Among the elastographic techniques, VCTE (FibroScan) has been the most extensively validated test for detecting and staging fibrosis. ${ }^{43,44}$ Suggested cutoffs for staging fibrosis are F0-F1 ( $<7 \mathrm{kPa}), \geq F 2(7-8.7 \mathrm{kPa}), \geq \mathrm{F} 3(8.7-10.3 \mathrm{kPa})$, and F4 $(\geq 10.3$ $\mathrm{kPa}) .{ }^{44}$ It may be difficult to obtain readings in obese individuals using the standard $\mathrm{M}$ probe. The availability of the $\mathrm{XL}$ probe has significantly improved the success rate with FibroScan in obese patients. ${ }^{45,46}$ The $\mathrm{XL}$ probe uses a lower frequency of ultrasound (2.5 MHz) when compared with the $\mathrm{M}$ probe (3.5 MHz). This allows the XL probe to carry out measurements at a greater depth of 35-75 $\mathrm{mm}$ when compared with $25-65 \mathrm{~mm}$ with the M probe. However, this lower frequency of ultrasound entails a lower velocity of the shear wave which may get interpreted as a lower liver stiffness measurement (LSM). Indeed, LSM values reported by the XL probe are slightly lower than that using the M probe and there has been much debate whether different cutoffs should be used for the XL probe. ${ }^{45}$ A recent prospective study of 496 patients with NAFLD suggested that similar cutoffs can be used for the two probes provided they are used according to the BMI (i.e., $\mathrm{M}$ probe for $\mathrm{BMI}<30 \mathrm{~kg} / \mathrm{m}^{2}$ and $\mathrm{XL}$ probe for $\mathrm{BMI} \geq 30 \mathrm{~kg} / \mathrm{m}^{2}$ ). ${ }^{46}$ The FibroScan device now incorporates an algorithm asking the operator to switch to the XL probe if it detects that the skin to liver capsule distance is $>25 \mathrm{~mm}$ or if the entered BMI of the patient is $\geq 30 \mathrm{~kg} / \mathrm{m}^{2}$. Point shear wave elastography and 2-D shear wave elastography allows localization of the region of interest as they can be mounted on conventional ultrasound machines. ${ }^{47}$ Furthermore, the readings are not affected by obesity. Although the cutoffs using these advanced elastographic techniques are expected to parallel those using FibroScan, there is little available real-world data in NAFLD. ${ }^{42,47}$ Blind extrapolation of cutoffs may not be clinically prudent and further studies are warranted. Newsome et al. recently derived the noninvasive FAST score for predicting the presence of NASH with significant fibrosis ( $Z F 2$ ) and inflammation (NAFLD activity score $\geq 4$ ) on biopsy. This score incorporates AST and the FibroScanderived parameters of CAP and LSM and was externally validated in multiple global cohorts. ${ }^{48}$

It is now recognized that $30-70 \%$ of cases of cryptogenic cirrhosis may be attributable to $\mathrm{NASH} .{ }^{17}$ Making the diagnosis of NASH in patients who have progressed to cirrhosis is often challenging. Evidence of NASH may be lacking on biopsy in these patients as steatosis tends to disappear with advancing fibrosis. The presence of two or more components of metabolic syndrome in the absence of other etiologies of liver disease supports the diagnosis of NASH in patients with cryptogenic cirrhosis. As weight loss is common in cirrhosis, past history of being overweight or obese should be considered as a risk factor while evaluating patients with NASH cirrhosis. ${ }^{17}$

\section{Management of Obese Patient with Nonalcoholic Fatty Liver Disease/ Nonalcoholic Steatohepatitis \\ General Management}

It is worthwhile to remember that the highest-burden of morbidity and mortality from NAFLD is attributed to cardiovascular complications. ${ }^{2,17}$ Statins are one of the most commonly prescribed drugs for decreasing the risk of cardiovascular complications due to improvement in atherogenic lipid profile. Despite initial concerns, statins have now been shown to be safe even in the presence of elevated transaminases. Improvement in insulin resistance and a possible decreased risk of HCC has also been reported with statins. ${ }^{49}$ Various other comorbidities are independently associated with both NAFLD and obesity like diabetes mellitus, dyslipidemia, polycystic ovarian disease, obstructive sleep apnea, osteoporosis, and chronic kidney disease. A holistic approach is thus of paramount importance., 2,17 Current evidence suggests that adequate management of metabolic risk factors may have additional hepatic benefits.

\section{Weight Loss}

Weight loss is the cornerstone in the management of NAFLD, particularly in obese patients. There is a direct correlation between the degree of weight loss and improvement in liver histology in NAFLD. Overall, a reduction in weight of 3-5\% may be sufficient for improvement in steatosis while a decrease of $>7-10 \%$ is required for improvement in steatohepatitis and fibrosis. ${ }^{50,51}$

\section{Lifestyle Interventions, Exercise, and Diet}

The combination of physical activity and dietary interventions in obese individuals results in a higher reduction in weight when compared with dietary intervention alone. ${ }^{52}$ Exercise also has a beneficial effect on NAFLD per se. In a large Korean study involving almost 2.3 lakh participants, the exercise of at least 10 minutes duration on $>5$ days in a week was associated with a significantly higher resolution of fatty liver on ultrasound at 5 years of follow-up. ${ }^{53}$ Evolving evidence suggests that exercise alone can lead to improvements in insulin resistance and possibly NAFLD even without weight loss. ${ }^{54}$ Although the data in NAFLD is limited, present evidence suggests that the magnitude of improvement in steatosis does not correlate with the intensity of exercise. ${ }^{55}$ An exercise schedule incorporating aerobic exercises for 45-60 minutes, at least 5 days a week to achieve a target heart rate of $60-70 \%$ of maximal heart rate is generally recommended. In obese individuals, brisk walking may be an ideal initial exercise. Duration should be tailored to the individual's tolerance levels and be gradually stepped up. Resistance training has been less extensively evaluated in NAFLD. In a systematic review evaluating 12 studies including 13 aerobic and 4 resistance training protocols, both aerobic and resistance exercises were found to improve steatosis. Although there was no difference in the duration of exercise required, resistance exercise was able to improve steatosis with significantly lower energy consumption. ${ }^{56}$ This suggests that resistance training may be particularly helpful in obese patients with poor cardiorespiratory fitness and those who are unable to tolerate or partake in aerobic exercises.

Benefits of exercise and increased energy expenditure risks being nullified unless accompanied by a decrease in energy intake. Calorie restriction should thus go hand-in-hand with exercise. A diet hypocaloric by $25-30 \%$ or $500-1,000 \mathrm{kcal} /$ day is generally recommended. In general, carbohydrates should account for $50-60 \%$ of the calorie intake while $25-30 \%$ of the calories are contributed by dietary fats with saturated fats being $<10 \%$ of the dietary fat intake. Extremely hypocaloric diets should be avoided as they are difficult to sustain and may be associated with a paradoxical worsening of liver enzymes. ${ }^{17}$

An association between sweetened beverages and hepatic steatosis was shown in a cross-sectional analysis of the 
Framingham Heart Study cohort. ${ }^{57}$ Increased severity of the liver disease has been observed with high fructose intake, although there is some evidence to the contrary. ${ }^{58}$ As such sugar-sweetened beverages particularly those containing fructose should be discouraged in these patients. Snacks in-between meals should also be avoided. There is much recent interest in intermittent fasting but its role in NAFLD needs further evaluation. Initial studies showed improvement in liver biochemistry with the use of omega-3-fatty acids. However, no benefit with regards to histological endpoints of NASH was seen in three placebocontrolled, randomized controlled trials of omega-3-fatty acids including ethyl-eicosapentaenoic, eicosapentaenoic acid, and docosahexaenoic acids. ${ }^{59-61}$

A lot of attention has recently been focused on the type of diet. The Mediterranean diet is rich in mono and poly unsaturated fatty acids, and aromatic compounds. It has been shown to decrease cardiovascular disease, cancer, obesity, type 2 diabetes mellitus (T2DM), and associated mortality. ${ }^{55}$ There is also some evidence of the beneficial effects of the Mediterranean diet in NAFLD. In two European studies from Spain and Greece, adherence to the Mediterranean diet was associated with a decreased risk of having NASH in patients with biopsy-proven NAFLD. ${ }^{62}$ Decreased hepatic steatosis at 6 months was observed using ultrasound in patients on a Mediterranean diet in a randomized controlled trial comprising 98 Italian patients with NAFLD. ${ }^{62}$ Furthermore, in a small cross-over study of 12 patients with biopsy-proven NAFLD, 6-week Mediterranean diet was associated with decreased insulin resistance and decreased hepatic steatosis on magnetic resonance spectroscopy. ${ }^{63}$ Thus, some authorities recommend the Mediterranean diet in NAFLD. Furthermore, prospective, randomized trials with larger sample sizes and histological endpoints are needed to draw firm conclusions in this regard. A high protein diet has also been shown to be helpful in weight reduction and maintenance in obese patients and is a reasonable option in obese patients with NAFLD. Overall, calorie restriction appears to be more important than the precise nature of the diet and its constituent macronutrients. ${ }^{17}$ The dietary plan can be adapted according to local cuisines and individual preferences.

\section{Pharmacotherapy and Bariatric Procedures for Weight Loss}

A combination of dietary modifications with exercise is the first line of management for reducing weight. However, with lifestyle interventions alone, weight reduction is difficult to attain and even more difficult to sustain. With time, physiologic adaptations decrease satiety, increase hunger, and limit energy expenditure, thereby recreating a scenario that promotes weight gain. Indeed only around $10 \%$ of patients can attain a weight loss of $>10 \%$ and only around $5 \%$ of them can sustain this over a period of 12 months. ${ }^{55}$ In patients who fail to control their weight with lifestyle interventions, additional approaches may be used depending upon the BMI. While deciding on these approaches, NAFLD should be considered as a comorbidity.

Pharmacotherapy for obesity may be considered in those with $\mathrm{BMI} \geq 27 \mathrm{~kg} / \mathrm{m}^{2}{ }^{64}$ Among the drugs approved for obesity, there are data on the use of liraglutide and orlistat in patients with NAFLD. In the phase II LEAN trial, liraglutide, a glucagon-like-peptide-1 (GLP-1) receptor agonist, was associated with improved NASH resolution and decrease the progression of fibrosis. Liraglutide also led to a decrease in weight by $8 \mathrm{~kg}$ in responders and almost $4 \mathrm{~kg}$ in non-responders over a duration of 48 weeks. In comparison, the reduction in weight in the placebo arm was a measly $0.6 \mathrm{~kg}$.
Another point to note is that the dose of liraglutide that is approved for obesity $(3 \mathrm{mg})$ is higher than the dose that was evaluated in the LEAN trial $(1.8 \mathrm{mg}) .{ }^{65}$ In a recent phase II study, semaglutide, another GLP-1 receptor agonist, led to the resolution of NASH in a significantly higher proportion of patients when compared with placebo. The average weight loss in patients who received $0.4 \mathrm{mg}$ of semaglutide was $13 \%$ compared with only $1 \%$ in those who received placebo. ${ }^{66}$ Orlistat is a pancreatic lipase inhibitor that decreases the absorption of dietary triglycerides and can result in a weight loss of $>5 \%$ in approximately $40 \%$ of patients. Several studies have shown improvement in transaminases with orlistat in NAFLD. However, in a double-blind randomized controlled trial in 52 patients of NAFLD, orlistat did not improve histological endpoints, although there was a significant reduction in serum transaminases. ${ }^{67}$

Bariatric surgery has been shown to improve liver histology in addition to improved survival and cardiovascular outcomes and can be considered in otherwise eligible obese patients with NAFLD $\left(B M I>35 \mathrm{~kg} / \mathrm{m}^{2}\right) .{ }^{64}$ Various surgical techniques have been reported in patients with obesity and NAFLD, including sleeve gastrectomy, adjustable gastric banding, and Roux-en-Y gastric bypass. In a recent systematic review and meta-analysis of 21 studies including 2,374 patients, bariatric surgery led to an improvement in steatosis, steatohepatitis, and fibrosis in almost 90, 60, and $30 \%$ patients, respectively. ${ }^{68}$ However, in the absence of a randomized controlled trial, bariatric surgery is still not recommended as the primary treatment for patients with NAFLD/NASH.

Endoscopy for metabolic diseases is a rapidly evolving field. Various endoscopic methods for weight loss have been described among which intragastric bariatric balloons have received the most attention. One of the problems with these balloons is that they can be left in situ for only a limited amount of time (6-24 months depending upon manufacturer) and there is a possibility of weight regain after removal of the balloon. This mandate repeated procedures with additional costs. Furthermore, none of the endoscopic techniques for weight loss have been evaluated in NAFLD. Endoscopic duodenal mucosal resurfacing (DMR) has been reported to improve the metabolic profile and hepatic indices in patients with T2DM. ${ }^{69}$ Pending further data, these techniques cannot be recommended for the treatment of NAFLD per se but may indeed be beneficial by promoting weight loss.

\section{Pharmacotherapy for Nonalcoholic Steatohepatitis}

Therapeutic options for the treatment of NASH are limited. In the landmark PIVENS trial, vitamin E decreased hepatic steatosis, inflammation, and ballooning with an improvement in NAS and NASH resolution in the PIVENS trial. ${ }^{70}$ Various other studies including meta-analyzes have shown that vitamin E improves liver biochemistry and histology including steatosis, inflammation, and ballooning but not fibrosis in patients with NASH. Current guidelines recommend vitamin E (800 IU/day) in non-diabetic patients with biopsy-proven NASH. ${ }^{2}$ Concerns regarding increased all-cause mortality have been disproved in a large meta-analysis incorporating 57 studies. $^{2}$

Pioglitazone, a peroxisome proliferator-activated receptorgamma (PPAR- $\gamma$ ) agonist, is the other drug that is recommended by International guidelines in biopsy-proven patients with NASH with or without diabetes. Although the primary endpoint of "improvement in NAS by 2 points with at least 1-point improvement in hepatocellular ballooning and 1-point improvement in either the lobular inflammation or steatosis score, and no increase in the fibrosis score" was not attained in the pioglitazone arm in the 
PIVENS trial, significantly more patients on pioglitazone achieved resolution of NASH when compared with placebo. ${ }^{70}$ However, pioglitazone may lead to weight gain which may be particularly problematic in obese patients. An increased risk of osteoporosis and prostate cancer has also been suggested. ${ }^{2}$

Saroglitazar is a dual PPAR- $\alpha / \gamma$ agonist with 1,000 times higher affinity for PPAR- $\alpha$ when compared with PPAR- $\gamma$. In phase II randomized controlled trial (EVIDENCES IV), saroglitazar (4 $\mathrm{mg} /$ day) significantly improved serum transaminases, hepatic steatosis, insulin resistance, and dyslipidemia in NAFLD patients. ${ }^{71}$ In the multicentric, phase III, EVIDENCES II study, 102 patients with biopsy-proven non-cirrhotic NASH were randomized in a 2:1 ratio to receive either saroglitazar or placebo. Significantly more patients on saroglitazar (52.3\%) attained a decrease in NAS by more than two points without worsening of fibrosis when compared with those on placebo (23.5\%; $p=0.04) .{ }^{72}$ Based on these data, saroglitazar has recently been approved by the Drug Controller General of India (DGCl) for use in patients with NASH and F1-3 fibrosis.

\section{Management of Obese Patient with Nonalcoholic Steatohepatitis-related Cirrhosis or hepatocellular carcinoma}

Despite being obese, patients with NASH cirrhosis often have sarcopenia. ${ }^{73}$ Calorie restriction of 500-1,000 kcal/day should be accompanied by adequate protein intake $(1.5 \mathrm{~g} / \mathrm{kg}$ of body weight) to achieve weight loss without compromising protein stores. ${ }^{74}$ Earlier studies had suggested that exercise may lead to worsening of portal pressures in cirrhotics. However, this has been contradicted by more recent findings. In the SportDiet study, which evaluated dietary interventions and exercises in patients with compensated cirrhosis and obesity, a reduction in body weight $>5 \%$ was seen in $52 \%$ of patients. A reduction in hepatic venous pressure gradient (HVPG) $>10 \%$ was also observed in $42 \%$ of patients without worsening of CTP or model for end-stage liver disease (MELD) scores. ${ }^{75}$ Overall, lifestyle interventions should be individualized to target a weight loss of $5-10 \%$ in obese cirrhotics. ${ }^{74}$ There is little experience with anti-obesity drugs in cirrhosis and as such, they should be avoided. Extreme vigilance is warranted with bariatric surgery in patients with cirrhosis as the mortality ranges from $1-2.5 \%$ in compensated cirrhosis to $16.3 \%$ in decompensated cirrhosis. ${ }^{68}$ If deemed necessary laparoscopic sleeve gastrectomy may be a safer option but should be attempted only in CTP A compensated cirrhotics. ${ }^{76}$ Endoscopic placement of intragastric balloons may be a reasonable option in these patients as was demonstrated in a small Indian study. ${ }^{77}$

Non-alcoholic steatohepatitis-specific therapies like pioglitazone, vitamin E, or saroglitazar are not recommended in those with underlying cirrhosis. Recent retrospective data suggest that vitamin $E$ and metformin may reduce the risk of decompensation with increased transplant-free survival, and metformin may also decrease the risk of HCC risk. ${ }^{78,79}$ However, in the absence of prospective trials, this cannot be recommended in routine clinical practice.

Management of complications of portal hypertension and HCC should be along the same lines as followed in other cirrhotics. Patients with decompensated cirrhosis and MELD score $>15$ should be considered for a liver transplant. Overall, post-transplant graft and patient survival in NASH are excellent and comparable to that in other etiologies. ${ }^{80}$ In obese patients, particular care should be taken to adequately evaluate cardiorespiratory fitness before proceeding for transplant. A meta-analysis of 13 studies suggested that BMI does not affect survival in a patient undergoing liver transplant. ${ }^{81}$ Nonetheless, most transplant centers avoid transplants in morbidly obese patients and advise weight loss before proceeding with the transplant.

\section{Future pharmacotherapies for Nonalcoholic Steatohepatitis}

Several new drugs for NASH are in the pipeline. We have already alluded to the GLP-1 receptor agonists, liraglutide, and semaglutide, which may have added beneficial effects with respect to weight loss. The biggest interest currently is with obeticholic acid, a farnesoid $X$ receptor agonist. An interim analysis of phase III REGENERATE study in NASH patients with stage 2 or 3 fibrosis showed that the primary outcome of $\geq 1$ stage improvement in fibrosis without worsening of NASH was significantly more common in patients treated with 25 $\mathrm{mg}(23.1 \%, p=0.0002)$ and $10 \mathrm{mg}(17.6 \%, p=0.045)$ of obeticholic acid compared with placebo (11.9\%). However, pruritus was seen in nearly half of the patients on $25 \mathrm{mg}$ of obeticholic acid with treatment discontinuation in $13 \% .{ }^{82}$ Obeticholic acid is also being evaluated in compensated cirrhosis (REVERSE trial). Phase III trials are currently underway in fibrotic NASH with cenicriviroc (AURORA trial) and resmetirom (MAESTRO-NASH trial). Selonsertib did not show any benefit in improving fibrosis in patients with NASH and bridging fibrosis or compensated cirrhosis in phase III STELLAR trials. ${ }^{83}$ The results of the phase III RESOLVE-IT trial evaluating elafibranor in NASH with F2 or F3 fibrosis were recently presented at The Liver Meeting, AASLD 2020. Elafibranor did not attain the primary endpoint of NASH resolution without worsening of fibrosis. Several other drugs are being evaluated in phase I and phase II trials including apical sodium-dependent bile acid transporter inhibitor (volixibat), pan-caspase inhibitor (emricasan), selective PPAR-a modulators (pemafibrate), non-bile acid FXR agonist (tropifexor), FGF-21, and FGF-19 analogs.

\section{Conclusion}

There is an intrinsic link between the two lifestyle diseases, obesity, and NAFLD. Obese individuals not only have a higher risk of NAFLD but are also at a higher risk of developing more severe liver disease (NASH, NASH-related cirrhosis, and HCC). Control of associated other metabolic risk factors and weight loss primarily with lifestyle interventions continues to remain the cornerstone in the management of both obesity and NASH. Anti-obesity drugs and bariatric procedures are not recommended per se for the treatment of NAFLD/NASH. Of the various drugs, vitamin E, pioglitazone, and saroglitazar are well studied and are indicated in select patients with NASH. Managing obese patients with NASH cirrhosis is a challenge and requires individualized care.

\section{References}

1. González-Muniesa P, Mártinez-González MA, Hu FB, et al. Obesity. Nat Rev Dis Primers 2017(1):3. DOI: 10.1038/nrdp.2017.34.

2. Chalasani N, Younossi Z, Lavine JE, et al. The diagnosis and management of nonalcoholic fatty liver disease: practice guidance from the American association for the study of liver diseases. Hepatology 2018;67(1):328-357. DOI: 10.1002/hep.29367.

3. Younossi ZM. Non-alcoholic fatty liver disease - a global public health perspective. J Hepatol 2019;70(3):531-544. DOI: 10.1016/j. jhep.2018.10.033.

4. Bedogni G, Miglioli L, Masutti F, et al. Prevalence of and risk factors for nonalcoholic fatty liver disease: the dionysos nutrition and liver study. Hepatology 2005;42(1):44-52. DOI: 10.1002/hep.20734. 
5. Younossi ZM, Koenig AB, Abdelatif $D$, et al. Global epidemiology of nonalcoholic fatty liver disease-meta-analytic assessment of prevalence, incidence, and outcomes. Hepatology 2016;64(1):73-84. DOI: 10.1002/hep.28431.

6. Duseja A, Chalasani N. Epidemiology and risk factors of nonalcoholic fatty liver disease (NAFLD). Hepatol Int 2013;7(S2):755-764. DOI: 10.1007/s12072-013-9480-x.

7. Ekstedt M, Franzén LE, Mathiesen UL, et al. Long-term follow-up of patients with NAFLD and elevated liver enzymes. Hepatology 2006;44(4):865-873. DOI: 10.1002/hep.21327.

8. Eslam M, Newsome PN, Sarin SK, et al. A new definition for metabolic associated fatty liver disease: an international expert consensus statement. J Hepatol 2020;73(1):202-209. DOI: 10.1016/j. jhep.2020.03.039.

9. Duseja A, Taneja S. Changing nomenclature from nonalcoholic fatty liver disease to metabolic dysfunction-associated fatty liver disease - not only premature but also confusing. J Clin Exp Hepatol 2020. DOI: 10.1016/j.jceh.2020.08.002.

10. Mitra S, De A, Chowdhury A. Epidemiology of non-alcoholic and alcoholic fatty liver diseases. TransI Gastroenterol Hepatol 2020;5:16. DOI: 10.21037/TGH.2019.09.08.

11. World Health Organisation. Obesity and overweight. Accessed November 24, 2020. https://www.who.int/news-room/fact-sheets/ detail/obesity-and-overweight.

12. di Cesare M, Bentham J, Stevens GA, et al. Trends in adult bodymass index in 200 countries from 1975 to 2014: a pooled analysis of 1698 population-based measurement studies with 19.2 million participants. The Lancet 2016;387(10026):1377-1396. DOI: 10.1016/ S0140-6736(16)30054-X.

13. Dai $H$, Alsalhe TA, Chalghaf N, et al. The global burden of disease attributable to high body mass index in 195 countries and territories, 1990-2017: an analysis of the Global burden of disease study. PLoS Med 2020;17(7). DOI: 10.1371/journal.pmed.1003198.

14. Fan JG, Kim SU, Wong VWS. New trends on obesity and NAFLD in Asia. J Hepatol 2017;67(4):862-873. DOI: 10.1016/j.jhep.2017. 06.003.

15. Pradeepa R, Anjana R, Joshi S, et al. Prevalence of generalized \& abdominal obesity in urban \& rural India- the ICMR-INDIAB study (phase-I) [ICMR-INDIAB-3]. Indian J Med Res 2015;142(2):139-150. DOI: 10.4103/0971-5916.164234

16. Sayiner M, Koenig A, Henry L, et al. Epidemiology of nonalcoholic fatty liver disease and nonalcoholic steatohepatitis in the United States and the rest of the world. Clin Liver Dis 2016;20(2):205-214. DOI: 10.1016/j.cld.2015.10.001.

17. Duseja A, Singh SP, Saraswat VA, et al. Non-alcoholic fatty liver disease and metabolic syndrome-position paper of the Indian national association for the study of the liver, endocrine society of India, Indian college of cardiology and Indian society of gastroenterology. J Clin Exp Hepatol 2015;5(1):51-68. DOI: 10.1016/j.jceh.2015.02.006.

18. Duseja A, Najmy S, Sachdev S, et al. High prevalence of non-alcoholic fatty liver disease among healthy male blood donors of urban India. JGH Open 2019;3(2):133-139. DOI: 10.1002/jgh3.12117.

19. Duseja A, Das A, Das R, et al. The clinicopathological profile of Indian patients with nonalcoholic fatty liver disease (NAFLD) is different from that in the west. Dig Dis Sci 2007;52(9):2368-2374. DOI: 10.1007/ s10620-006-9136-y.

20. Duseja A, Das A, Dhiman RK, et al. Indian patients with nonalcoholic fatty liver disease presenting with raised transaminases are different at presentation. World J Gastroenterol 2007;13(4):649-650. DOI: 10.3748/wjg.v13.i4.649.

21. Madan K, Batra Y, Gupta SD, et al. Non-alcoholic fatty liver disease may not be a severe disease at presentation among Asian Indians. World J Gastroenterol:WJG 2006;12(21):3400. DOI: 10.3748/WJG.V12. 121.3400.

22. Rastogi A, Shasthry SM, Agarwal A, et al. Non-alcoholic fatty liver disease - histological scoring systems: a large cohort singlecenter, evaluation study. APMIS 2017;125(11):962-973. DOI: 10.1111/ apm.12742.
23. Schuster $S$, Cabrera D, Arrese $M$, et al. Triggering and resolution of inflammation in NASH. Nat Rev Gastroenterol Hepatol 2018;15(6):349364. DOI: $10.1038 / \mathrm{s} 41575-018-0009-6$.

24. De A, Duseja A. Natural history of simple steatosis or nonalcoholic fatty liver. J Clin Exp Hepatol 2020;10(3):255-262. DOI: 10.1016/j. jceh.2019.09.005.

25. Fabbrini E, Sullivan S, Klein S. Obesity and nonalcoholic fatty liver disease: biochemical, metabolic, and clinical implications. Hepatology 2010;51(2):679-689. DOI: 10.1002/hep.23280.

26. Stefan N, Häring HU, Schulze MB. Metabolically healthy obesity: the low-hanging fruit in obesity treatment? Lancet Diabetes Endocrinol 2018;6(3):249-258. DOI: 10.1016/S2213-8587(17)30292-9.

27. Young S, Tariq R, Provenza J, et al. Prevalence and profile of nonalcoholic fatty liver disease in lean adults: Systematic review and meta-analysis. Hepatol Communicat 2020;4(7):953-972. DOI: 10.1002/ hep4.1519.

28. Lonardo A, Mantovani A, Lugari S, et al. Epidemiology and pathophysiology of the association between NAFLD and metabolically healthy or metabolically unhealthy obesity. Ann Hepatol 2020;19(4):359-366. DOI: 10.1016/j.aohep.2020.03.001.

29. Ouchi N, Parker JL, Lugus JJ, et al. Adipokines in inflammation and metabolic disease. Nat Rev Immunol 2011;11(2):85-97. DOI: 10.1038/ nri2921.

30. de Heredia FP, Gómez-Martínez S, Marcos A. Chronic and degenerative diseases: obesity, inflammation and the immune system. In: Proceedings of the Nutrition Society, vol. 71. Cambridge University Press; 2012. pp. 332-338. DOI: 10.1017/S0029665112000092.

31. Hiuge-Shimizu A, Kishida K, Funahashi T, et al. Absolute value of visceral fat area measured on computed tomography scans and obesity-related cardiovascular risk factors in large-scale Japanese general population (the VACATION-J study). Ann Med 2012;44(1):8292. DOI: $10.3109 / 07853890.2010 .526138$.

32. Virtue S, Vidal-Puig A. It's not how fat you are, it's what you do with it that counts. PLoS Biol 2008;6(9):1819-1823. DOI: 10.1371/journal. pbio.0060237.

33. Pellegrinelli V, Carobbio S, Vidal-Puig A. Adipose tissue plasticity: how fat depots respond differently to pathophysiological cues. Diabetologia 2016;59(S):1075-1088. DOI: 10.1007/s00125-016-3933-4.

34. Stefan N, Schick F, Häring HU. Causes, characteristics, and consequences of metabolically unhealthy normal weight in humans. Cell Metab 2017;26(2):292-300. DOI: 10.1016/j.cmet.2017.07.008.

35. Lotta LA, Gulati P, Day FR, et al. Integrative genomic analysis implicates limited peripheral adipose storage capacity in the pathogenesis of human insulin resistance. Nat Genet 2017;49(1):17-26. DOI: 10.1038/ ng.3714.

36. Misra A, Misra R, Wijesuriya M, et al. The metabolic syndrome in South Asians: continuing escalation and possible solutions. Indian J Med Res 2007;125(3):345-354.

37. Ross R, Neeland IJ, Yamashita S, et al. Waist circumference as a vital sign in clinical practice: a consensus statement from the IAS and ICCR working group on visceral obesity. Nat Rev Endocrinol 2020;16(3):177-189. DOI: 10.1038/s41574-019-0310-7.

38. Saadeh S, Younossi ZM, Remer EM, et al. The utility of radiological imaging in nonalcoholic fatty liver disease. Gastroenterology 2002;123(3):745-750. DOI: 10.1053/gast.2002.35354.

39. Karlas T, Petroff $D$, Sasso M, et al. Individual patient data metaanalysis of controlled attenuation parameter (CAP) technology for assessing steatosis. J Hepatol 2017;66(5):1022-1030. DOI: 10.1016/j. jhep.2016.12.022.

40. Dulai PS, Singh S, Patel J, et al. Increased risk of mortality by fibrosis stage in nonalcoholic fatty liver disease: systematic review and metaanalysis. Hepatology 2017;65(5):1557-1565. DOI: 10.1002/hep.29085.

41. Manuel Echevarría J, León P, Pozo F, et al. EASL-ALEH clinical practice guidelines: non-invasive tests for evaluation of liver disease severity and prognosis. J Hepatol 2015;63(1):237-264. DOI: 10.1016/j. jhep.2015.04.006.

42. Wong VWS, Adams LA, de Lédinghen V, et al. Noninvasive biomarkers in NAFLD and NASH — current progress and future promise. Nat Rev 
Gastroenterol Hepatol 2018;15(8):461-478. DOI: 10.1038/s41575-0180014-9.

43. Kwok R, Tse Y-K, Wong GL-H, et al. Systematic review with metaanalysis: non-invasive assessment of non-alcoholic fatty liver disease - the role of transient elastography and plasma cytokeratin-18 fragments. Aliment Pharmacol Ther 2014;39(3):254-269. DOI: 10.1111/ apt.12569.

44. Wong VWS, Vergniol J, Wong GLH, et al. Diagnosis of fibrosis and cirrhosis using liver stiffness measurement in nonalcoholic fatty liver disease. Hepatology 2010;51(2):454-462. DOI: 10.1002/hep.23312.

45. Wong VWS, Vergniol J, Wong GLH, et al. Liver stiffness measurement using XL probe in patients with nonalcoholic fatty liver disease. Am J Gastroenterol 2012;107(12):1862-1871. DOI: 10.1038/ajg.2012.331.

46. Wong VWS, Irles M, Wong GLH, et al. Unified interpretation of liver stiffness measurement by $M$ and $X L$ probes in non-alcoholic fatty liver disease. Gut 2019;68(11):2057-2064. DOI: 10.1136/gutjnl-2018-317334.

47. Friedrich-Rust M, Poynard T, Castera L. Critical comparison of elastography methods to assess chronic liver disease. Nat Rev Gastroenterol Hepatol 2016;13(7):402-411. DOI: 10.1038/ nrgastro.2016.86.

48. Newsome PN, Sasso M, Deeks JJ, et al. FibroScan-AST (FAST) score for the non-invasive identification of patients with non-alcoholic steatohepatitis with significant activity and fibrosis: a prospective derivation and global validation study. Lancet Gastroenterol Hepatol 2020;5(4):362-373. DOI: 10.1016/S2468-1253(19)30383-8

49. Dongiovanni P, Petta S, Mannisto V, et al. Statin use and non-alcoholic steatohepatitis in at risk individuals. J Hepatol 2015;63(3):705-712. DOI: 10.1016/j.jhep.2015.05.006.

50. Vilar-Gomez E, Martinez-Perez Y, Calzadilla-Bertot L, et al. Weight loss through lifestyle modification significantly reduces features of nonalcoholic steatohepatitis. Gastroenterology 2015;149(2):367-378. e5. DOI: 10.1053/j.gastro.2015.04.005.

51. Glass LM, Dickson RC, Anderson JC, et al. Total body weight loss of $\geq 10 \%$ is associated with improved hepatic fibrosis in patients with nonalcoholic steatohepatitis. Dig Dis Sci 2015;60(4):1024-1030. DOI: 10.1007/s10620-014-3380-3.

52. Wu T, Gao X, Chen M, et al. Long-term effectiveness of diet-plusexercise interventions vs. Diet-only interventions for weight loss: a meta-analysis: obesity management. Obes Rev 2009;10(3):313-323. DOI: 10.1111/j.1467-789X.2008.00547.x.

53. Sung KC, Ryu S, Lee JY, et al. Effect of exercise on the development of new fatty liver and the resolution of existing fatty liver. J Hepatol 2016;65(4):791-797. DOI: 10.1016/j.jhep.2016.05.026.

54. Schweitzer GG, Klein S. Exercise and NAFLD: Is it worth the effort? Hepatology 2017;66(5):1691-1694. DOI: 10.1002/hep.29356.

55. Younossi Z, Loomba R, Rinella $M$, et al. Current and future therapeutic regimens for Non-alcoholic fatty liver disease (NAFLD) and nonalcoholic steatohepatitis (NASH). Hepatology 2017;777(5):1-36. DOI: 10.1002/hep.

56. Hashida R, Kawaguchi T, Bekki M, et al. Aerobic vs. Resistance exercise in non-alcoholic fatty liver disease: a systematic review. J Hepatol 2017;66(1):142-152. DOI: 10.1016/j.jhep.2016.08.023.

57. Ma J, Fox CS, Jacques PF, et al. Sugar-sweetened beverage, diet soda, and fatty liver disease in the Framingham heart study cohorts. J Hepatol 2015;63(2):462-469. DOI: 10.1016/j.jhep.2015.03.032.

58. Abdelmalek MF, Suzuki A, Guy C, et al. Increased fructose consumption is associated with fibrosis severity in patients with nonalcoholic fatty liver disease. Hepatology 2010;51(6):1961-1971. DOI: 10.1002/hep.23535.

59. Scorletti E, Bhatia L, Mccormick KG, et al. Effects of purified eicosapentaenoic and docosahexaenoic acids in nonalcoholic fatty liver disease: results from the WELCOME* study. Hepatology 2014;60(4):1211-1221. DOI: 10.1002/hep.27289.

60. Sanyal AJ, Abdelmalek MF, Suzuki A, et al. No significant effects of ethyl-eicosapentanoic acid on histologic features of nonalcoholic steatohepatitis in a phase 2 triaL. Gastroenterology 2014;147(2). DOI: 10.1053/j.gastro.2014.04.046.
61. Argo CK, Patrie JT, Lackner C, et al. Effects of $n-3$ fish oil on metabolic and histological parameters in NASH: A double-blind, randomized, placebo-controlled trial. J Hepatol 2015;62(1):190-197. DOI: 10.1016/j. jhep.2014.08.036.

62. Brunner KT, Henneberg CJ, Wilechansky RM, et al. Nonalcoholic fatty liver disease and obesity treatment. Curr Obes Rep 2019;8(3):220-228. DOI: 10.1007/s13679-019-00345-1.

63. Ryan MC, Itsiopoulos C, Thodis T, et al. The mediterranean diet improves hepatic steatosis and insulin sensitivity in individuals with non-alcoholic fatty liver disease. J Hepatol 2013;59(1):138-143. DOI: 10.1016/j.jhep.2013.02.012.

64. Chitturi S, Wong VW-S, Chan W-K, et al. The Asia-pacific working party on Non-alcoholic fatty liver disease guidelines 2017-part 2: management and special groups. J Gastroenterol Hepatol 2018;33(1):86-98. DOI: 10.1111/jgh.13856.

65. Armstrong MJ, Gaunt P, Aithal GP, et al. Liraglutide safety and efficacy in patients with non-alcoholic steatohepatitis (LEAN): a multicentre, double-blind, randomised, placebo-controlled phase 2 study. The Lancet 2016;387(10019):679-690. DOI: 10.1016/S01406736(15)00803-X.

66. Newsome PN, Buchholtz K, Cusi K, et al. A placebo-controlled trial of subcutaneous semaglutide in nonalcoholic steatohepatitis. N Eng J Med, Published online November 13 2020. NEJMoa2028395. DOI: 10.1056/NEJMoa2028395.

67. Zelber-Sagi S, Kessler A, Brazowsky E, et al. A double-blind randomized placebo-controlled trial of orlistat for the treatment of nonalcoholic fatty liver disease. Clin Gastroenterol Hepatol 2006;4(5):639-644. DOI: 10.1016/j.cgh.2006. 02.004 .

68. Fakhry TK, Mhaskar R, Schwitalla T, et al. Bariatric surgery improves nonalcoholic fatty liver disease: a contemporary systematic review and meta-analysis. Surg Obes Relat Dis 2019;15(3):502-511. DOI: 10.1016/j.soard.2018.12.002.

69. van Baar ACG, Beuers U, Wong K, et al. Endoscopic duodenal mucosal resurfacing improves glycaemic and hepatic indices in type 2 diabetes: 6-month multicentre results. JHEP Reports 2019;1(6):429437. DOI: 10.1016/j.jhepr.2019.10.006.

70. Sanyal AJ, Chalasani N, Kowdley KV, et al. Pioglitazone, vitamin E, or placebo for nonalcoholic steatohepatitis. N Eng J Med 2010;362(18):1675-1685. DOI: 10.1056/nejmoa0907929.

71. Sarin S, Sharma M, Koradia P, et al. A prospective, multi-center, double-blind, randomized trial of saroglitazar $4 \mathrm{mg}$ versus placebo in patients with non-alcoholic steatohepatitis (EVIDENCES II). Hepatol Int 2020;14(s1):S326.

72. Gawrieh S, Loo NM, Mohseni R, et al. A phase 2, prospective, multicenter, double-blind, randomized study of saroglitazar magnesium $1 \mathrm{mg}, 2 \mathrm{mg}$ or $4 \mathrm{mg}$ versus placebo in patients with nonalcoholic fatty liver disease and/or nonalcoholic steatohepatitis (EVIDENCES IV). Hepatology 2019;70(s6):1484-1485A.

73. Carias S, Castellanos AL, Vilchez V, et al. Nonalcoholic steatohepatitis is strongly associated with sarcopenic obesity in patients with cirrhosis undergoing liver transplant evaluation. J Gastroenterol Hepatol (Australia) 2016;31(3):628-633. DOI: 10.1111/jgh.13166.

74. Merli M, Berzigotti A, Zelber-Sagi S, et al. EASL clinical practice guidelines on nutrition in chronic liver disease. J Hepatol 2019;70(1):172-193. DOI: 10.1016/j.jhep.2018.06.024.

75. Berzigotti A, Albillos A, Villanueva C, et al. Effects of an intensive lifestyle intervention program on portal hypertension in patients with cirrhosis and obesity: the SportDiet study. Hepatology 2017;65(4):1293-1305. DOI: 10.1002/hep.28992.

76. Rebibo L, Gerin O, Verhaeghe P, et al. Laparoscopic sleeve gastrectomy in patients with NASH-related cirrhosis: a case-matched study. Surg Obes Relat Dis 2014;10(3):405-410. DOI: 10.1016/j.soard.2013. 09.015 .

77. Choudhary NS, Puri R, Saraf N, et al. Intragastric balloon as a novel modality for weight loss in patients with cirrhosis and morbid obesity awaiting liver transplantation. Indian J Gastroenterol 2016;35(2):113116. DOI: $10.1007 / \mathrm{s} 12664-016-0643-2$. 
78. Vilar-Gomez E, Vuppalanchi R, Desai AP, et al. Long-term metformin use may improve clinical outcomes in diabetic patients with nonalcoholic steatohepatitis and bridging fibrosis or compensated cirrhosis. Aliment Pharmacol Therapeut 2019;50(3):317-328. DOI: 10.1111/apt.15331.

79. Vilar-Gomez E, Vuppalanchi R, Gawrieh S, et al. Vitamin E improves transplant-free survival and hepatic decompensation among patients with nonalcoholic steatohepatitis and advanced fibrosis. Hepatology 2020;71(2):495-509. DOI: 10.1002/hep.30368.

80. Haldar D, Kern B, Hodson J, et al. Outcomes of liver transplantation for non-alcoholic steatohepatitis: a European liver transplant registry study. J Hepatol 2019;71(2):313-322. DOI: 10.1016/j.jhep.2019.04.011.
81. Saab $S$, Lalezari $D$, Pruthi $P$, et al. The impact of obesity on patient survival in liver transplant recipients: a meta-analysis. Liver Int 2015;35(1):164-170. DOI: 10.1111/liv.12431.

82. Younossi Z, Ratziu V, Loomba R, et al. GS-06-positive results from REGENERATE: a phase 3 international, randomized, placebocontrolled study evaluating obeticholic acid treatment for NASH. J Hepatol 2019;70(1):e5. DOI: 10.1016/S0618-8278(19) 30006-4.

83. Harrison SA, Wong VWS, Okanoue T, et al. Selonsertib for patients with bridging fibrosis or compensated cirrhosis due to NASH: results from randomized phase III STELLAR trials. J Hepatol 2020;73(1):26-39. DOI: 10.1016/j.jhep.2020.02.027.. 